\title{
ON THE DESIGN OF LYAPUNOV FUNCTIONS FOR STATE-DEPENDENT IMPULSIVE DYNAMICAL SYSTEMS
}

\author{
L. Burlion ${ }^{*, * *}$, T. Ahmed-Ali ${ }^{* * *}$ and F. Lamnabhi-Lagarrigue ${ }^{*}$ \\ * LSS-CNRS, SUPELEC, 3 Rue Joliot Curie \\ 91192 Gif-sur-Yvette, France \\ ** DGA-LRBA, BP 914 \\ 27207 Vernon Cedex, France \\ *** ENSIETA 2 Rue François Verny \\ 29806 Brest Cedex 9, France
}

\begin{abstract}
In this paper, we use viability tools to design Lyapunov functions for nonlinear state-dependent impulsive dynamical systems : the main idea is to partition the continuous state space. The approach enables to address fundamental issues in Hybrid systems such as accessibility and controllability. Copyright (c)2005 IFAC
\end{abstract}

Keywords: Nonlinear systems, Hybrid systems, Lyapunov methods

\section{INTRODUCTION}

There exist several papers dealing with the concept of Lyapunov function in the context of hybrid systems. See for instance, (Branicky, 1998), (Ye, Michel and Hou, 1998), (Chellaboina, Bhat and Haddad, 2003) or more recently (Goebel, Hespanha, Teel, Cai and Sanfelice, 2004). Although more restrictions have been assumed (see for instance (Ye, Michel and Hou, 1998+)), the first and most simple idea is the following one : in the context of hybrid systems, the Lyapunov function, which is discontinuous at the resetting times, has a negative derivative outside the resetting times (as usual) and decreases at the resetting times. The main disadvantage of such characterization is that it enables to check if a given function $\mathrm{V}$ is a Lyapunov function for an impulsive system but it does not give the way to design the Lyapunov function. In the classical (non hybrid) case, the problem is the same: it is not always possible to design Lyapunov functions but it is possible to design Lyapunov functions and controllers for specific classes of non linear systems (see for instance
(Krstic, Kanellakopoulos and Kokotovic, 1995)): however, it seems difficult to extend such ideas in the hybrid case since the system is submitted to discrete inputs (jumps, changes of dynamics). One way to try to recover the known techniques is to "catch the global behavior" of the hybrid system by partitioning the state space.

Viability theory is particularly well adapted for this purpose : (in the case of switching systems, see for instance (Burlion, Ahmed-Ali and Lamnabhi-Lagarrigue, 2004)). As we shall see (thanks to an introductive example), partitioning the state space is the crucial point to design a Lyapunov function for an hybrid impulsive system and we here propose to use viability theory to solve this problem.

The paper is organized as follows: we first present the class of system we address. We then give an introductive example to present the problem. Afterwards, we provide the reader with some mathematical tools before proving our results. We conclude by numerical computations and additional remarks. 


\section{MOTIVATION}

First, let us consider the following class of impulsive dynamical systems

$$
\left\{\begin{array}{l}
\dot{x}(t)=f(x(t)) \quad, \quad x(t) \notin J \\
\Delta x(t)=x\left(t^{+}\right)-x\left(t^{-}\right), \quad x\left(t^{-}\right) \in J
\end{array}\right.
$$

where $\mathrm{J}$ (the jumping set) is a subset of the state space. For example,

$$
\begin{aligned}
\dot{x} & = \begin{cases}-x & , \quad x \in]-\infty,-2[\cup[-1,+\infty[ \\
x & , \quad x \in]-2,-1[\end{cases} \\
\triangle x(t) & =\frac{3}{2} \quad, \quad x(t)=-2
\end{aligned}
$$

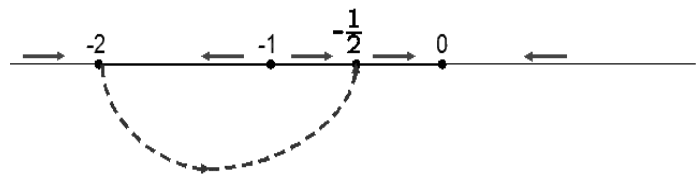

Fig. 1. An example of an impulsive system

It is easy to check that starting from any initial point, there is at most one switching time and the following Lyapunov function satisfies the classical theorems. ( $\dot{V}$ decreases almost everywhere and $\mathrm{V}$ is discontinuous and decreases at the switching points)

$$
V(x)=\left\{\begin{array}{l}
x^{2}, \quad x \in[-1,+\infty[ \\
|x+2|+1, \quad x \in]-\infty,-1[
\end{array}\right.
$$

The idea is the following one: starting from any point of ] $-\infty,-1$ [ we go in finite time toward -2 because it is the point from which it is possible to jump into the "good interval" which is $[-1,+\infty[$. We call it "good" because this is the interval which contains 0 and on which we can use the classical tools (we mean non hybrid) to design a Lyapunov function. Therefore, the design of the Lyapunov function consists of

i) designing a Lyapunov function (valid on $[-1,+\infty[)$ as usually

ii) designing another Lyapunov function (valid on ] $-\infty,-1[$ ) which enables to reach -2 in finite time. (This means this Lyapunov function cancels at the point -2 )

iii) concatenating these functions

In this paper, we are going to consider the control of impulsive systems of the form

$$
\left\{\begin{array}{c}
\dot{x}(t)=f\left(x(t), u_{c}(t)\right), \quad x(t) \notin J \\
\Delta x(t)=r\left(x\left(t^{-}\right), u_{d}\left(t^{-}\right)\right)-x\left(t^{-}\right) \\
x\left(t^{-}\right) \in J
\end{array}\right.
$$

As illustrated in the above example, the idea is here to identify also for this class of systems, the "good" region of the state space which contains the origin and then the regions from which we can jump into the good "one". Then, the design of the Lyapunov function will consist of

i) partitioning the state space

ii) choosing a Lyapunov function in the "good" region

iii) extending the first function by finding other Lyapunov functions which cancel on the switching points and tend to 0 in finite time.

In the next parts we are going to see not only that viability theory is an adapted tool that enables us to find a partition of the state space but also that it allows to obtain (numerically) the Lyapunov functions on each region.

Indeed, the above class of controlled impulsive systems (2) can be reformulate by setting

$$
\begin{aligned}
& F(x):=\left\{f\left(x, u_{c}\right) ; u_{c} \in \mathcal{U}\right\} \\
& R(x):=\left\{r\left(x, u_{d}\right) ; u_{d} \in \mathcal{V}\right\}
\end{aligned}
$$

Then, we deal with following equivalent impulsive differential inclusion

$$
\left\{\begin{array}{l}
\dot{x}(t) \in F(x(t)), \quad x(t) \notin J \\
x\left(t^{+}\right) \in R\left(x\left(t^{-}\right)\right), \quad x\left(t^{-}\right) \in J
\end{array}\right.
$$

The control law is hybrid in the sense it has discrete and continuous components: the discrete control law $u_{d}$ is a discrete event input which acts on the reset value of the state at a jump. The continuous control law $u_{c}$ is defined as usually. When $u_{c}$ (resp. $u_{d}$ ) belongs to a set $\mathcal{U}$ (resp. $\mathcal{V}$ ), this amounts to consider differential inclusions.

Before showing our main result, let us recall some mathematical backgrounds on differential inclusions and on impulsive differential inclusion (3).

\section{MATHEMATICAL BACKGROUND AND NOTATIONS}

In the present paper, we are concerned with finite-dimensional dynamical systems with statedependent impulse effects. Here, the state space is a normed vectorial space $(X,\|\|$.$) (usually, X$ is $\mathbb{R}^{n}$ ). $\mathrm{d}$ denotes the distance associated to the norm $\|$.$\| .$

Given $(X, d), \bar{A}$ and $\partial A$ denote the closure and the boundary of the subset $A \subset X$. Given two subsets $\mathrm{B}$ and $\mathrm{C}, B \backslash C$ is the set of elements of $\mathrm{B}$ which do not belong to $\mathrm{C}$. Evolutions are continuous functions $x():. t \in \mathbb{R}^{+} \mapsto x(t) \in X$ describing the evolution of the state $x(t)$ of a dynamical system. 
The basic definitions dealing with set-valued maps also called multivalued functions or correspondences can be found in (Aubin, 1991)(chap.2).

The graph $\operatorname{Graph}(F)$ of a set-valued map $F$ : $\mathbb{R}^{n} \longrightarrow 2^{\mathbb{R}^{n}}$ (usually noted $F: \mathbb{R}^{n} \rightsquigarrow \mathbb{R}^{n}$ ) is the set of pairs $(x, y) \in \mathbb{R}^{n} \times \mathbb{R}^{n}$ satisfying $y \in F(x)$. The domain of $F, \operatorname{Dom}(F)$ is the subset of elements $x \in \mathbb{R}^{n}$ such that $F(x)$ is not empty.

$F$ is called lower semi-continuous at $x \in \mathbb{R}^{n}$ if for all $y \in F(x)$ and for all sequences $x_{n}$ converging to $x$, there exists a sequence $y_{n} \in F\left(x_{n}\right)$ converging to $y$. The inverse image of a subset $K \subset X$ under a set-valued map $R: X \rightsquigarrow X$ is defined by

$$
R^{-1}(K):=\{x \in X, \quad R(x) \cap K \neq \emptyset\}
$$

We say that a set-valued map $\mathrm{F}$ is Marchaud if

- $\operatorname{Graph}(F)$ and $\operatorname{Dom}(F)$ are non empty and closed

- the values $F(x)$ of $F$ are convex

- the growth of $\mathrm{F}$ is linear i.e $\exists c>0$ such that

$$
\begin{gathered}
\forall x \in X, \\
\|F(x)\|:=\sup _{v \in F(x)}\|v\| \leq c(\|x\|+1)
\end{gathered}
$$

The epigraph of a function $V: X \longrightarrow \mathbb{R}$ is defined by

$$
\operatorname{Epi}(V):=\{(x, y) \in X \times \mathbb{R}, \quad V(x) \leq y\}
$$

Let us consider the following differential inclusion

$$
\dot{x}(t) \in F(x(t))
$$

where $\mathrm{F}$ is Marchaud.

A solution to this differential inclusion starting at $x_{0} \in X$ is an absolutely continuous function $x: \mathbb{R}^{+} \longrightarrow X$ such that $x(0)=x_{0}$ and almost everywhere $\dot{x}(t) \in F(x(t))$. For any $x_{0} \in \mathbb{R}^{n}$, $\mathcal{S}\left(x_{0}\right)$ denotes the set of evolutions $x($.$) governed$ by (4) starting from $x_{0}$.

Let $K, \quad C \subset X$. The viability kernel of $K$ with target $C$ under $\mathcal{S}$ is the subset $\operatorname{Viab}_{\mathcal{S}}(K, C)$ of initial states $x_{0} \in K$ such that (at least) one evolution $x(.) \in \mathcal{S}\left(x_{0}\right)$ is viable in $K$ for all $t \geq 0$ or viable in $K$ until it reaches $C$ in finite time.

$\operatorname{Viab}_{\mathcal{S}}(K, C)=\left\{x_{0} \in K, \exists T \geq 0, \exists x(.) \in \mathcal{S}\left(x_{0}\right)\right.$, $\forall t \in \mathbb{R}^{+}, \quad x(t) \in K \quad$ or $\forall t \in[0, T[, \quad x(t) \in K$ and $x(T) \in C\}$

When the target $C=\emptyset$, it is said that $\operatorname{Viab}_{\mathcal{S}}(K)=$ $\operatorname{Viab}_{\mathcal{S}}(K, \emptyset)$ is the viability kernel of $\mathrm{K}$. The capture basin of $C$ viable in $K$ under $\mathcal{S}$ is the subset $\operatorname{Capt}_{\mathcal{S}}(K, C)$ of initial states $x_{0} \in K$ such that (at least) one evolution $x(.) \in \mathcal{S}\left(x_{0}\right)$ is viable in $\mathrm{K}$ until it reaches $\mathrm{C}$ in finite time.

$\operatorname{Capt}_{\mathcal{S}}(K, C)=\left\{x_{0} \in K, \exists T \geq 0, \exists x(.) \in \mathcal{S}\left(x_{0}\right)\right.$, $\forall t \in[0, T[, \quad x(t) \in K$ and $x(T) \in C\}$
Let us now consider the class of dynamical systems of the form

$$
\left\{\begin{array}{l}
\dot{x}(t) \in F(x(t)), x(t) \notin J \\
x\left(t^{+}\right) \in R\left(x\left(t^{-}\right)\right), x\left(t^{-}\right) \in J
\end{array}\right.
$$

where

- $x\left(t^{-}\right) \triangleq \lim _{s \longrightarrow t^{-}} x(s)$

- the set $\stackrel{s \longrightarrow t^{-}}{\subset}$ is compact

- $F X \rightsquigarrow X$ is Marchaud

- $R X \rightsquigarrow X$ is upper semi continuous with compact images we call it the reset map

- $R$ also verifies $R(\partial J) \cap J=\emptyset$

The collection $(F, R, J)$ is called an impulse differential inclusion. Throughout this paper, we only interest us to evolutions starting outside $J$. This means that all the initial conditions belong to the complement of the subset $J$ in $X$. The state $x$ is instantaneously reset when it belongs to the jumping set $J$. We note $\tau_{1}, \ldots, \tau_{k}, \ldots$ the reset times. In the hybrid terminology, an evolution $x($. is defined in the following way:

Definition: (see (Aubin, Lygeros, Quincampoix, Sastry and Seube, 2002), or (Cruck, 2002)) $x($.) : $\mathbb{R}^{+} \longrightarrow X$ is a solution of the above impulse differential inclusion if and if only it is absolutely continuous on some intervals $\left[\tau_{k}, \tau_{k+1}\right.$ [ having leftside limits, with $\left(\tau_{k}\right)_{k \in \mathbb{N}^{*}}$ an increasing finite or infinite sequence of $\mathbb{R}^{+*} \cup\{+\infty\}$ such that

$$
x(0)=x_{0} \in X \backslash J
$$

and

$$
x(t) \in\left\{x_{0}\right\}+\sum_{\tau_{k} \leq t} S\left(x\left(\tau_{k}\right)\right)+\int_{0}^{t} F(x(s)) d s
$$

where

$$
S(x)=\{z \in X / \exists y \in R(x), z=y-x\} .
$$

Let $\mathcal{C}_{m}$ denotes the set of right-continuous functions having left-side limits. We note

$$
S: \mathbb{R}^{n} \backslash J \longrightarrow \mathcal{C}_{m}\left(\mathbb{R}^{+}, \mathbb{R}^{n}\right)
$$

the evolution solution map associated with the impulsive dynamical system.

Remark: The condition $R(\partial J) \cap J=\emptyset$ guarantees that there is a lower bound on the transition times i.e $\forall k \in \mathbb{N}^{*}$, such that $\tau_{k}$ is finite,

$$
\tau_{k+1}-\tau_{k}>0
$$

Proof of the remark : By definition, since F is Marchaud, there exists $c \in \mathbb{R}^{+}$s.t $: \forall x \in$ $X,\|F(x)\| \leq c(\|x\|+1)$.

Thus, $\forall k \in \mathbb{N}^{*}, \forall t \in\left[\tau_{k}, \tau_{k+1}[\right.$ 


$$
\begin{aligned}
\left\|x(t)-x\left(\tau_{k}\right)\right\| \leq & \int_{\tau_{k}}^{t}\|F(x(y))\| d y \\
\leq & \int_{\tau_{k}}^{t} c(\|x(y)\|+1) d y \\
\leq & \int_{\tau_{k}}^{t} c\left(\left\|x\left(\tau_{k}\right)\right\|+1\right. \\
& \left.+\left\|x(y)-x\left(\tau_{k}\right)\right\|+1\right) d y
\end{aligned}
$$

By Gronwall's inequality

$\left\|x(t)-x\left(\tau_{k}\right)\right\| \leq c\left(\tau_{k+1}-\tau_{k}\right)\left(1+\left\|x\left(\tau_{k}\right)\right\|\right) e^{c\left(\tau_{k+1}-\tau_{k}\right)}$

Since, $R(\partial J)$ and $J$ are closed the condition $R(\partial J) \cap J=\emptyset$ yields

$$
\exists \delta>0, \quad \forall(x, y) \in J \times R(\partial J), \quad\|x-y\|>\delta
$$

Thus,

$$
c\left(\tau_{k+1}-\tau_{k}\right)\left(1+\left\|x\left(\tau_{k}\right)\right\|\right) e^{c\left(\tau_{k+1}-\tau_{k}\right)}>\delta
$$

Hence, $\tau_{k+1}-\tau_{k}>0 . \square$

\section{MAIN RESULTS}

\subsection{Preliminaries}

Statement of the problem: We consider a compact subset $K \subset X$ such that $J \subset K$. For $\varepsilon>0$ fixed, we also consider $B_{\varepsilon}=\left\{x \in X, \quad d\left(x, 0_{X}\right) \leq \varepsilon\right\}$. We suppose that

- $B_{\varepsilon} \cap J=\emptyset$

- $B \subset K$

- $B_{\varepsilon}$ is not a repeller i.e $\operatorname{Viab}_{\mathcal{S}}\left(B_{\varepsilon}\right) \neq \emptyset$

We notice that $\operatorname{Viab}_{\mathcal{S}}\left(B_{\varepsilon}\right)$ is defined for a "non impulsive differential inclusion" because $B_{\varepsilon} \cap J=$ $\emptyset$.

The problem we consider here is to reach the target $B_{\varepsilon}$ and to deduce function which enjoys a Lyapunov property (the terminology will be defined further) for hybrid impulsive systems.

\subsection{Partition of the state-space}

Let us denote $R(\partial J)$ by $\mathcal{R}_{J}$ and $\operatorname{Viab}_{\mathcal{S}}\left(B_{\varepsilon}\right)$ by $\partial J_{0}$. We now recursively define the following sets

$$
C_{0}=\operatorname{Capt}_{S}\left(K \backslash J, \partial J_{0}\right)
$$

and

$\forall i \geq 1, \quad C_{i}=\operatorname{Capt}_{S}\left((K \backslash J) \cup \partial J_{i}, \partial J_{i} \cup_{j<i} C_{j}\right)$

where

$$
\partial J_{i}=\left(\partial J \cap R^{-1}\left(C_{i-1} \cap \mathcal{R}_{J}\right)\right) \backslash\left(\cup_{j<i} \partial J_{j}\right) .
$$

We obtain the following result:

Theorem: $\forall i \in \mathbb{N}^{*}$ fixed, $\forall x_{0} \in C_{i}$, there exists at least one evolution starting from $x_{0}$ which jumps at most $i$ times and then is stabilized in a neighboring of 0 in finite time.

Proof: The algorithm first computes $C_{0}$. By definition, $C_{0}=\operatorname{Capt}_{\mathcal{S}}\left(K \backslash J, \partial J_{0}\right)$ is the set of all initial states (of $\mathrm{K}$ ) from which it is possible to stay in $K \backslash J$ (so without any jumps) before reaching the target in finite time. To extend the set of initial points from which one can reach the target, we then consider that the state can jump one time in this case, this amounts to searching for initial conditions from which it is possible to reach $\mathrm{J}$ in finite time and then to jump in $C_{0}$. Then, the target becomes the intersection of the inverse image of $C_{0}$ by $R$ and $\partial J$. We also add to the target the previous set $C_{0}$ we get $C_{1}$ which is the set of initial conditions from which it is possible to reach the target $\partial J_{0}$ in finite time without jumping more than 1 time (more precisely, if the initial condition is in $C_{1} \backslash C_{0}$ it exactly jumps 1 time). We repeat the following procedure to take into account two jumps and so on...

From now on, we are only interested with a finite number $\mathrm{N}$ of jumps. This means that the "working space" will be

$$
\Omega=\bigcup_{i \in\{0, \cdots, N\}} C_{i}
$$

4.3 Resulting control strategy for controlled impulsive systems

Let us now consider again the control of impulsive systems (2) of the form

$$
\left\{\begin{array}{c}
\dot{x}(t)=f\left(x(t), u_{c}(t)\right), \quad x(t) \notin J \\
\Delta x(t)=r\left(x\left(t^{-}\right), u_{d}\left(t^{-}\right)\right)-x\left(t^{-}\right) \\
x\left(t^{-}\right) \in J
\end{array}\right.
$$

One can easily check that if

- $\mathrm{f}$ is continuous in both variables, affine with respect to $\mathrm{u}$, has linear growth, and $\mathcal{U}$ is nonempty convex and compact.

- $\mathrm{r}$ is continuous, and $\mathcal{V}$ is nonempty and compact

the reformulation of system (2) into an impulsive differential equation is such that $\mathrm{F}$ is Marchaud and $\mathrm{R}$ is upper semi continuous with compact images. In this case, one can apply the previous result.

Theorem: Starting from any initial condition of $\Omega \subset K$, the hybrid controller

$$
\forall i \in\{1, \cdots, N\}, \quad \forall x \in C_{i} \backslash\left(\partial J_{i} \cup_{j<i} C_{j}\right),
$$

$$
u_{c}(x) \in U_{C_{i}} \quad(x)
$$

$\forall x \in \partial J_{i}$

$$
u_{d}(x) \in\left\{v \in \mathcal{V} \backslash r(x, v) \in C_{i-1}\right\}
$$


$\forall x \in V i a b_{\mathcal{S}}\left(B_{\varepsilon}\right)$,

$$
u_{c}(x) \in R_{\operatorname{iab}_{\mathcal{S}}\left(B_{\varepsilon}\right)} \quad(x)
$$

where $R_{K}(x)$ denotes the set of viable controls associated to each element $x$ of a given viability domain $K$ (for more details, see (Aubin, 1991), chap 6 and $7, R_{K}$ is given by the viability theorem) and where $U_{C}(x)$ denotes the set of controls associated to each element $x$ of a given capture basin $C$ $\left(U_{C}\right.$ is given by the numerical computation of the capture basin which is computed thanks to the viability kernel of an augmented system (for numerical methods, see (Saint-Pierre, 1994))) enables the state $x$ to reach the viability kernel $\operatorname{Viab}_{\mathcal{S}}\left(B_{\varepsilon}\right)$ in finite time without jumping more than $N$ times. Then, the state remains inside this kernel.

Proof: This theorem is a direct application of the previous theorem. Indeed, the state space has been partitioned such that inside each capture basin $C_{i}$ there exists at least one feedback which enables to reach $\partial J_{i}$ and to jump into the next capture basin $C_{i-1}$ and so on, until the state reaches the target $C_{0}=\operatorname{Viab}_{\mathcal{S}}\left(B_{\varepsilon}\right)$. The discrete feedback is simply chosen such that the state jumps into $C_{i-1}$ when $x \in \partial J_{i}$ and the continuous feedback is given numerically in each capture basins. Inside the target $\operatorname{Viab}_{\mathcal{S}}\left(B_{\varepsilon}\right)$, the use of viable feedbacks (given by the viability theorem due to (Ye, Michel and Hou, 1998) ) obviously prevents the state from escaping it.

\subsection{Design of a Lyapunov Function}

Another application of the partition of the state space is the design of Lyapunov functions for impulsive systems.

Taking into account state constraints while searching for a Lyapunov function amounts to giving up some smooth properties of the Lyapunov function by using viability tools, we obtain Lyapunov functions which are no longer continuous but lower semi continuous ; moreover, they are no longer differentiable but contingently epidifferentiable.

Let us explain a little more what we mean by Lyapunov functions. We will say that the nontrivial extended function

$$
V: X \longrightarrow \mathbb{R}^{+} \cup\{+\infty\}
$$

enjoys a Lyapunov property if and if only for any initial state $x_{0}$, there exists at least one solution $x($.$) to the impulsive differential inclusion (??)$ satisfying

$$
\forall t \geq 0, \quad V(x(t)) \leq y(t)
$$

where the time dependent function $y \mathbb{R} \longrightarrow \mathbb{R}^{+} \cup$ $\{+\infty\}$ is governed by the ordinary differential equation

$$
\dot{y}(t)=\phi(y(t))
$$

where $\phi$ is continuous with linear growth. In many cases, $y(t)$ goes to 0 when $t \longrightarrow+\infty$, so $\mathrm{V}(\mathrm{x}(\mathrm{t}))$ converges also to 0 . For more details, see (Aubin, 1991)(Chap.9).

We consider a finite number $N \in \mathbb{N}$ which represents the maximum number of jumps. Let us consider

$$
\left\{\begin{array}{l}
\dot{x}(t) \in F(x(t)) \quad, x(t) \in C_{0} \\
\dot{y}(t)=-a y(t)
\end{array}\right.
$$

where $a \in \mathbb{R}^{+*}$

We also consider $\forall i \in\{1, \cdots, N\}$

$$
\left\{\begin{array}{l}
\dot{x}(t) \in F(x(t)) \quad, x(t) \in C_{i} \\
\dot{y}(t)=\phi(y(t))
\end{array}\right.
$$

where $\phi: \mathbb{R} \longrightarrow \mathbb{R}$ is continuous with linear growth (in this case, one can easily check that

$$
\left(\begin{array}{c}
F \\
\phi
\end{array}\right) X \times \mathbb{R} \rightsquigarrow X \times \mathbb{R}
$$

is Marchaud ) such that $\forall y(0)>0, y(t)=0$ in finite time. For instance, we choose $\phi=-1$.

By noting

$$
\begin{gathered}
v_{i}(x)=d\left(x, \partial J_{i}\right) \quad, x \in C_{i} \\
E_{p}\left(v_{i}\right)=\left\{(x, y) \in C_{i} \times \mathbb{R}^{+} \backslash v_{i}(x) \leq y\right\}
\end{gathered}
$$

gives

Theorem: The extended nontrivial function $V$ such that

$$
\begin{aligned}
\operatorname{Dom}(V) & =\underset{i \in\{0, \cdots, N\}}{\bigcup} C_{i} \\
\lambda_{v_{i}}(x) & =\inf _{(x, y) \in \operatorname{Viab}_{(10)} E_{p}\left(v_{i}\right)} y \\
V(x) & =\lambda_{v_{i}}(x) \quad, x \in C_{i} \backslash\left(\cup_{j<i} C_{j}\right) .
\end{aligned}
$$

exponentially decreases after a finite time $T \geq 0$ which depends on the initial condition $x(0)$.

\section{Proof:}

Step 1:

Given $x(0)$ we first search for $\mathrm{k}$ such that $x(0) \in$ $C_{k}$ and $x(0) \notin C_{k-1}$. In this case, $\mathrm{x}$ stays in $C_{k} \backslash C_{k-1}$ until it reaches $\partial J_{k}$ and jumps. (if it reached $C_{k-1}$ without jumping before, there would be a contradiction because it would prove that $x(0) \in C_{k-1}$ since it would be possible to reach it without jumping). We call $\tau_{k}$ the jumping time. By definition of the epigraph, for $t \in\left[0, \tau_{k}\right]$, $V(x()$.$) verifies$

$$
0 \leq d\left(x(t), \partial J_{k}\right) \leq V(x(t)) \leq y(t)
$$

Moreover, to say that $(x, y)$ belongs to the viability kernel of $E_{p}\left(v_{k}\right)$ under auxiliary system (10) 
for all $t \in\left[0, \tau_{k}\right]$ amounts to saying that for all $t \in\left[0, \tau_{k}\right]$,

$$
0 \leq d\left(x(t), \partial J_{k}\right) \leq V(x(t)) \leq y(t)
$$

with

$$
\begin{aligned}
y(t) & =y(0)+\int_{0}^{t} \phi(y(\tau)) d \tau \\
& =y(0)-t
\end{aligned}
$$

Then, $\lim _{t \longrightarrow \tau_{k}^{-}} V(x(t))=0$. At $t=\tau_{k}, \mathrm{~V}$ is discontinuous and $\mathrm{x}$ jumps into $C_{k-1}$. We then set $t=0$ and repeat the step 1 until we jump into $C_{0}$.

Step 2:

At a time $\tau, \mathrm{x}$ belongs to $C_{0}$. To say that $(x, y)$ belongs to the viability kernel of $E_{p}\left(v_{0}\right)$ under auxiliary system (9) at the time $t=\tau$ amounts to saying that for all $t \geq \tau$,

$0 \leq d\left(x(t), \partial J_{0}\right) \leq V(x(t)) \leq y(t)=y(\tau) e^{-a(t-\tau)}$

which is the property of an exponential lyapunov function.

\section{Step 3: conclusion of the proof}

We then designed a function $\mathrm{V}$ which decreases to 0 . First, according to the initial condition, $\mathrm{V}$ may decrease to zero and jump a finite number of times (at most $\mathrm{N}$ times). After a finite time, $\mathrm{x}$ belongs to $C_{0}$ and the function $\mathrm{V}$ decreases exponentially to 0 .

\section{CONCLUSION}

In this paper, we presented a new approach to design controllers and Lyapunov functions for impulsive dynamical systems. This new approach relies heavily on Viability theory which is a powerful tool in control its main drawback is the numerical difficulty of computing the capture basins and viability kernels for high-dimensional systems. To combine "more classical" control techniques with viability when numerical computations are too huge could also be a key to control such systems for instance, viability could be use to partition the state space and one could use other techniques to deduce the control laws inside the different basins since the extraction of the control laws with given properties requires computation in higher dimensional state spaces.

\section{ACKNOWLEDGEMENT}

The authors are very thankful to Pr. Jean-Pierre Aubin for several constructive comments.

\section{REFERENCES}

J.P. Aubin (1991). "Viability Theory", Birkhauser, Boston, Basel, Berlin.

J.P. Aubin and H. Frankowska (1990). "SetValued Analysis", Birkhauser, Boston, Basel, Berlin.

J.P. Aubin, J. Lygeros, M.Quincampoix, S.Sastry and N. Seube (2002). "Impulse Differential Inclusions A viability approach to hybrid systems", IEEE Transactions on Automatic Control, vol.47, no.1, pp 2-20.

E. Cruck (2002), "Target Problems under State Constraints for Nonlinear Controlled Impulsive Systems ", J.Math.Anal.Appl. 270, pp 636-656.

P. Saint-Pierre (1994), "Approximation of the Viability Kernel”, Appl.Math.Optim. 29, pp 187-209.

L. Burlion, T. Ahmed-Ali and F. LamnabhiLagarrigue (2004), "Stabilization of Hybrid Switching Systems a Viability Approach", in Proc. of MTNS 2004, Leuven, Belgium.

M.S. Branicky (1998), "Multiple Lyapunov Functions and Other Analysis Tools for Switched and Hybrid Systems", IEEE Transactions on Automatic Control, vol.43, no.4, pp 475-482.

H. Ye, A.N. Michel and L. Hou (1998), "Stability Theory for Hybrid Dynamical Systems", IEEE Transactions on Automatic Control, vol.43, no.4, pp 461-474.

H. Ye, A.N. Michel and L. Hou (1998+), "Stability Analysis of Systems with Impulse Effects", IEEE Transactions on Automatic Control, vol.43, pp 1719-1723.

V. Chellaboina, S.P. Bhat and W.M. Haddad (2003), "An Invariance Principle for Nonlinear Hybrid and Impulsive Dynamical Systems", Nonlinear Analysis, 53, pp 527-550.

R. Goebel, J. Hespanha, A. Teel, C. Cai and R. Sanfelice (2004), "Hybrid Systems Generalized Solutions and Robust Stability", In Proc. of NOLCOS 2004, Stuttgart, Germany.

M. Krstic, I. Kanellakopoulos and P. Kokotovic (1995), "Nonlinear and Adaptative Control Design", Wiley-Interscience, New York, 